\title{
The Impact of Systematic Review Automation Tools on Methodological Quality and Time Taken to Complete Systematic Review Tasks: Case Study
}

Justin Clark $^{1}$, BA; Catherine McFarlane ${ }^{2,3}, \mathrm{PhD}$; Gina Cleo ${ }^{1}$, PhD; Christiane Ishikawa Ramos ${ }^{2,4}, \mathrm{PhD}$; Skye Marshall ${ }^{2,5}$, $\mathrm{PhD}$

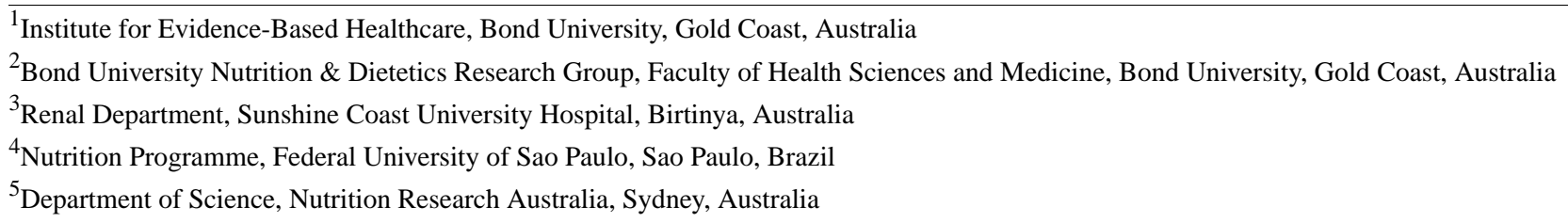

Corresponding Author:

Justin Clark, BA

Institute for Evidence-Based Healthcare

Bond University

Building 5, Level 4

14 University Drive, Robina

Gold Coast, 4226

Australia

Phone: 610755855545

Email: jclark@bond.edu.au

\section{Abstract}

Background: Systematic reviews (SRs) are considered the highest level of evidence to answer research questions; however, they are time and resource intensive.

Objective: When comparing SR tasks done manually, using standard methods, versus those same SR tasks done using automated tools, (1) what is the difference in time to complete the SR task and (2) what is the impact on the error rate of the SR task?

Methods: A case study compared specific tasks done during the conduct of an SR on prebiotic, probiotic, and synbiotic supplementation in chronic kidney disease. Two participants (manual team) conducted the SR using current methods, comprising a total of 16 tasks. Another two participants (automation team) conducted the tasks where a systematic review automation (SRA) tool was available, comprising of a total of six tasks. The time taken and error rate of the six tasks that were completed by both teams were compared.

Results: The approximate time for the manual team to produce a draft of the background, methods, and results sections of the SR was 126 hours. For the six tasks in which times were compared, the manual team spent 2493 minutes (42 hours) on the tasks, compared to 708 minutes (12 hours) spent by the automation team. The manual team had a higher error rate in two of the six tasks-regarding Task 5: Run the systematic search, the manual team made eight errors versus three errors made by the automation team; regarding Task 12: Assess the risk of bias, 25 assessments differed from a reference standard for the manual team compared to 20 differences for the automation team. The manual team had a lower error rate in one of the six tasks-regarding Task 6: Deduplicate search results, the manual team removed one unique study and missed zero duplicates versus the automation team who removed two unique studies and missed seven duplicates. Error rates were similar for the two remaining compared tasks—regarding Task 7: Screen the titles and abstracts and Task 9: Screen the full text, zero relevant studies were excluded by both teams. One task could not be compared between groups-Task 8: Find the full text.

Conclusions: For the majority of SR tasks where an SRA tool was used, the time required to complete that task was reduced for novice researchers while methodological quality was maintained.

(JMIR Med Educ 2021;7(2):e24418) doi: 10.2196/24418 


\section{KEYWORDS}

systematic reviews; automation; technology assessment; methods evaluation; case study; comparison study

\section{Introduction}

\section{Overview}

Health care guidelines have reported systematic reviews (SRs) as providing the highest level of evidence to answer research questions [1]. The findings of SRs are favored as they synthesize all published evidence on a topic in a rigorous, reproducible, and transparent way [2]. SRs are used to answer any type of research question, including interventional, diagnostic, prognostic, or etiological [1]; in addition, they are pertinent to many different stakeholders' groups, from clinicians to researchers to policy makers. However, SRs are time and resource intensive [3] and may be out of date by the time they are published [4]. The time from SR registration to publication has been reported as taking five authors approximately 67 weeks [5], with time frames ranging from 6 months to 2 years [6]. Even rapid reviews, which omit some of the steps of a full SR, have been reported to take 7 to 12 months [7].

To improve time to completion, systematic review automation (SRA) tools have been developed to either fully automate or semi-automate one or more specific tasks involved in conducting an SR. These include searching multiple databases [8], deduplicating search results [9], identifying disagreements between screeners [10,11], and assessing risk of bias (RoB) in randomized controlled trials (RCTs) [12]. In 2015, the International Collaboration for the Automation of Systematic Reviews (ICASR) was formed to enable resource sharing between groups developing SRA tools [13].

However, despite SRA tool availability, the tools have not been translated into practice, primarily due to distrust of the tools [14]. This may be caused by a lack of transparency of machine learning systems and a shortage of studies evaluating the SRA tools [15]. The third ICASR meeting in 2017 identified the need to overcome barriers to SRA uptake [16]. A potential solution is to evaluate SRA tools in a real-world setting, on real SRs, to test their performance. This case study was designed to do that in the health care field of chronic kidney disease.

\section{Research Questions}

When comparing SR tasks done manually, using standard methods, versus those same SR tasks done using SRA tools, (1) what is the difference in time to complete the SR task and (2) what is the impact on the error rate of the SR task?

\section{Methods}

A case study on the methods used to undertake an SR of RCTs delivering a health care intervention was conducted and has been reported according to the STROBE (Strengthening the Reporting of Observational Studies in Epidemiology) statement [17].

\section{Ethics Approval and Consent to Participate}

Ethics approval was not sought; all participants are authors on this manuscript and the SR tasks undertaken were in an SR in which ethical approval was not required.

\section{Case Study Participants}

An expression of interest was sent to the Bond University Faculty of Health Sciences and Medicine, Australia, seeking researchers planning to commence an SR of RCTs. The only group to volunteer had their SR used in this case study. The SR was conducted by a team of four researchers using current Cochrane methodology [2] and reported using PRISMA (Preferred Reporting Items for Systematic Reviews and Meta-Analyses) guidelines [18]. Two of these researchers (CM and CR) were novice researchers completing their first SR under the supervision of two experienced researchers who were not involved in this case study. These two novice researchers (CM and $\mathrm{CR}$ ) were sampled as the participants on the manual team.

A second expression of interest was sent to the faculty seeking two other researchers not involved in the SR to comprise the automation team. This expression was sent to researchers in the same discipline (ie, nutrition and dietetics) to ensure sufficient knowledge of the SR topic. The only interested candidates (SM and GC) took on the role of the participants on the automation team. As new postdoctoral researchers, they had some experience of being part of an SR team (Table 1).

Table 1. Characteristics of study participants' roles and experience.

\begin{tabular}{lllll}
\hline $\begin{array}{l}\text { Team and participants } \\
\text { (initials) }\end{array}$ & Team role & Research role & $\begin{array}{l}\text { Coauthor of completed SRs }{ }^{\mathrm{a}} \\
\text { (eg, middle author), } \mathrm{n}\end{array}$ & $\begin{array}{l}\text { Lead author of completed SRs }^{\mathrm{a}} \\
(\mathrm{eg}, \text { first author), } \mathrm{n}\end{array}$ \\
\hline $\begin{array}{l}\text { Manual team } \\
\text { CM }\end{array}$ & Primary & PhD student & 0 & 0 \\
CR & Secondary & PhD student & 0 & 0 \\
$\begin{array}{l}\text { Automation team } \\
\text { SM }\end{array}$ & Primary & Postdoctoral researcher & 0 & 3 \\
GC & Secondary & Postdoctoral researcher & 1 & 0 \\
\hline
\end{tabular}

${ }^{\text {a }}$ SR: systematic review; published, accepted for publication, or under review. 


\section{Case Study Systematic Review}

The SR used in this study-Prebiotic, probiotic, and synbiotic supplementation in chronic kidney disease: A systematic review and meta-analysis-has been published [19]. To complete the SR, four databases were searched, 717 results were deduplicated, 596 titles and abstracts were screened for inclusion, 16 studies were included, and 10 studies were meta-analyzed (Table 2).

Table 2. Characteristics of the completed and published systematic reviews (SRs) [19].

\begin{tabular}{lll}
\hline SR task & SR task description & Value, $\mathrm{n}$ \\
\hline Run the SR & Databases searched & 4 \\
Run the SR & Trial registries searched & 2 \\
Deduplicate the search results & Records to be deduplicated & 717 \\
Deduplicate the search results & Records left after deduplication & 586 \\
Screen the titles and abstracts & Studies to screen & 586 \\
Find the full text & Full texts required & 40 \\
Screen the full text & Full texts for screening & 40 \\
Extract the data & Full-text articles extracted (ie, characteristics of studies and outcomes) & 16 \\
Assess the risk of bias & Full-text articles requiring risk-of-bias assessment & 16 \\
Write the results & Full-text articles qualitatively synthesized \\
Conduct a meta-analysis & Full-text articles meta-analyzed & 16 \\
\hline
\end{tabular}

\section{The Systematic Review Tasks Conducted in the Study}

The manual team conducted the SR tasks required to complete a draft of the background, methods, and results sections of the SR; in total, this comprised 16 SR tasks (Table 3 [8,9,12,20,21]) [22]. The automation team conducted the tasks that had an SRA tool available; this comprised six SR tasks. Where an SR task is normally done by a single investigator, such as deduplicating search results, it was done by a single participant-the primary researcher-on each team. Where an SR task is normally done by two people, such as screening the search results, it was done by two participants - the primary and secondary researchers - on each team. 
Table 3. List and evaluation criteria of all systematic review (SR) tasks and systematic review automation (SRA) tools used.

\begin{tabular}{|c|c|c|c|}
\hline $\begin{array}{l}\text { SR task } \\
\text { No. }\end{array}$ & SR task & SRA tool used & Evaluation criteria \\
\hline 1 & Formulate the question & $\mathrm{N} / \mathrm{A}^{\mathrm{a}}$ & N/A \\
\hline 2 & Check for similar reviews & N/A & N/A \\
\hline 3 & Write the protocol & N/A & N/A \\
\hline 4 & Design the systematic search & N/A & N/A \\
\hline 5 & Run the systematic search & Polyglot Search Translator [8] & $\begin{array}{l}\text { Completed by one participant; the number of different types of errors } \\
\text { were counted. }\end{array}$ \\
\hline 6 & Deduplicate the search results & Deduplicator [9] & $\begin{array}{l}\text { Completed by one participant; deduplicated EndNote libraries were } \\
\text { compared to a deduplicated reference standard data set. }\end{array}$ \\
\hline 7 & Screen the titles and abstracts & SRA-Helper $^{\mathrm{b}}[20]$ & $\begin{array}{l}\text { Completed by two participants; EndNote libraries of the included } \\
\text { and excluded studies were compared. A wrongfully excluded study } \\
\text { was considered an error. }\end{array}$ \\
\hline 8 & Find the full text & $\begin{array}{l}\text { EndNote, SRA-Helper [20], } \\
\text { and SARA }{ }^{\mathrm{c}}[21]\end{array}$ & $\begin{array}{l}\text { Completed by one participant; the number of references ordered } \\
\text { through the library was compared. }\end{array}$ \\
\hline 9 & Screen the full text & SRA-Helper [20] & $\begin{array}{l}\text { Completed by two participants; EndNote libraries of the included } \\
\text { and excluded studies were compared. }\end{array}$ \\
\hline 10 & Conduct a citation analysis & N/A & N/A \\
\hline 11 & Extract the data & N/A & N/A \\
\hline 12 & Assess the risk of bias & RobotReviewer [12] & $\begin{array}{l}\text { Completed by two participants; the risk-of-bias assessments were } \\
\text { compared to a reference standard created by two experienced sys- } \\
\text { tematic reviewers external to the two teams. }\end{array}$ \\
\hline 13 & Synthesize the data & N/A & N/A \\
\hline 14 & Rerun the systematic search & N/A & N/A \\
\hline 15 & Conduct a meta-analysis & N/A & N/A \\
\hline 16 & Write the results & N/A & N/A \\
\hline
\end{tabular}

${ }^{\mathrm{a}}$ N/A: not applicable; this task did not have any relevant SRA tools.

${ }^{\mathrm{b}}$ SRA-Helper: Systematic Review Accelerator Helper.

${ }^{\mathrm{c}}$ SARA: System for Automatically Requesting Articles.

\section{The Systematic Review Automation Tools Used in the Study}

The decision-making framework used to select the five SRA tools used in this study considered the following: (1) tools that were freely (ie, no cost) available for use, (2) tools that were familiar to the experienced author (JC) in order to aid the participants, (3) availability of help guides, and (4) tools that could be applied to as many tasks as possible.

Polyglot Search Translator [8] was selected to automatically translate search strings between various health databases. Deduplicator was selected to detect duplicate records from the search results, allowing the user to view them and then select which ones to keep and which to discard. The Systematic Review Accelerator Helper (SRA-Helper) was selected to interface with EndNote to enable assignment to groups (ie, screening) using a hot key (eg, the space bar), thereby replacing the normal drag-and-drop method used when screening in EndNote. SRA-Helper was also used to help find the full text by interfacing with EndNote to enable hot keys to conduct a title search for articles in a set of predetermined locations: the Bond University Library catalog, PubMed, and Google Scholar. The System for Automatically Requesting Articles (SARA) was selected to interface directly with the Bond University Library system to request up to 500 full texts at a time with a single click. The fifth and final tool used was the RobotReviewer tool [12]. This tool allows users to upload the PDF of an RCT; it will then provide an RoB assessment in four of the seven domains of the Cochrane Collaboration's RoB tool [23]: random sequence generation, allocation concealment, blinding of participants and researchers, and blinding of outcome assessment (Table 4). 
Table 4. Systematic review automation (SRA) tools used in this study.

\begin{tabular}{|c|c|c|c|}
\hline $\mathrm{SR}^{\mathrm{a}}$ task No. & SR task & SRA tool used & SRA tool description \\
\hline 5 & Run the systematic search & $\begin{array}{l}\text { Polyglot Search Translator } \\
\text { [8] }\end{array}$ & $\begin{array}{l}\text { This tool translates searches from either a PubMed or Ovid MEDLINE } \\
\text { search string into a search string that can be used in multiple other } \\
\text { databases. }\end{array}$ \\
\hline 6 & Deduplicate the search results & Deduplicator [9] & $\begin{array}{l}\text { This tool allows the uploading of sets of references; it then detects and } \\
\text { removes duplicate references. }\end{array}$ \\
\hline 7 & Screen the titles and abstracts & SRA-Helper ${ }^{\mathrm{b}}[20]$ & $\begin{array}{l}\text { This is an automation script used to move references into groups within } \\
\text { EndNote software using a predetermined set of keyboard shortcuts. }\end{array}$ \\
\hline 8 & Find the full text & $\begin{array}{l}\text { SRA-Helper [20] and } \\
\text { SARA }^{\mathrm{c}}[21]\end{array}$ & $\begin{array}{l}\text { SRA-Helper is an automation script used to search predefined locations, } \\
\text { such as library websites, PubMed, and Google Scholar. SARA is a tool } \\
\text { that allows for the bulk requesting of articles (ie, document delivery) } \\
\text { from an institutional library. }\end{array}$ \\
\hline 9 & Screen the full text & SRA-Helper [20] & $\begin{array}{l}\text { This is an automation script used to move references into groups within } \\
\text { EndNote software using a predetermined set of keyboard shortcuts. }\end{array}$ \\
\hline 12 & Assess the risk of bias (RoB) & RobotReviewer [12] & $\begin{array}{l}\text { This is a machine learning system that automatically assesses RoB for } \\
\text { four of the seven domains defined by the Cochrane Collaboration's } \\
\text { RoB tool; it also highlights the supporting text for these assessments. }\end{array}$ \\
\hline
\end{tabular}

${ }^{\mathrm{a}} \mathrm{SR}$ : systematic review.

${ }^{\text {b }}$ SRA-Helper: Systematic Review Accelerator Helper.

${ }^{\mathrm{c}}$ SARA: System for Automatically Requesting Articles.

\section{Outcomes}

The outcomes recorded and compared were (1) the time taken to complete each task (in minutes) and (2) the error rate for each task (count).

\section{Comparison of Outcomes Between Teams}

For the single-participant SR tasks (ie, run the systematic search, deduplicate the search results, and find the full text), the primary manual team participant (CM) was compared to the primary automation team participant (SM). For the dual-participant SR tasks (ie, screen the titles and abstracts, screen the full text, and assess the RoB), the time and errors of the primary and secondary participants on each team were added together.

\section{Time Taken for the Systematic Review Tasks}

The time taken for each SR task was recorded separately for (1) undertaking the SR task and (2) learning about the SR task. Learning about each SR task included discussion with experts, reading help guides, or watching help videos. Time was recorded by each individual participant by noting the time they started work on the SR task and noting the time they finished work on the SR task. The total time spent on each task was calculated by subtracting the start time from the finish time. If a task was split over several work sessions, participants added together the times for each work session for each task to give the total time. Timing was paused if the participants foresaw a delay of 5 minutes or longer. The recording of times by the manual team began at Task 5: Run the systematic search. Times reported before this were retrospective estimates made by the participants.

\section{Measuring the Methodological Quality of Each Systematic Review Task}

Methodological quality was measured by the number of errors each team made for each SR task. As most SR tasks, as well as errors made during task performance, differ substantially, so did the way we evaluated each SR task.

\section{Evaluation of Systematic Review Task 5: Run the Systematic Search}

The systematic search was evaluated by counting the number of different types of errors made during the translation process. The errors were determined by a Cochrane information specialist and health librarian (David Honeyman; see Acknowledgments) with over 10 years' experience. The librarian was blinded as to which team had done the translations. Error criteria are listed in Table S1 in Multimedia Appendix 1.

\section{Evaluation of Systematic Review Task 6: Deduplicate the Search Results}

The deduplicated EndNote libraries were compared to a reference standard data set. This reference standard was created and the comparison made by an experienced information specialist (JC). This reference standard was created blind prior to the results from the manual and automation teams being made available. Any unique studies removed and the number of duplicates missed were recorded as errors.

\section{Evaluation of Systematic Review Tasks 7 and 9: Screen the Titles and Abstracts and Screen the Full Text}

EndNote libraries of the studies after screening and dispute resolution from both teams were compared by an experienced information specialist. An incorrectly excluded study was considered an error. The total number of references that were included and moved to the next task (ie, obtain full text) was also recorded. Any incorrectly excluded studies were sent to the senior author on the published SR, who did not participate in this case study. 


\section{Evaluation of Systematic Review Task 8: Find the Full Text}

Both teams ran the EndNote Find Full Text feature. Once this was completed and EndNote had automatically found as many full texts as it could, the teams attempted to find the remaining ones. This is when the evaluation between teams started. The number of references that were not found and had to be ordered through the library was the evaluation criterion. However, due to differences in institutional access by participants, the results of this evaluation were not reported.

\section{Evaluation of Systematic Review Task 12: Assess the} Risk of Bias

An RoB reference standard was created by two experienced systematic reviewers: an experienced information specialist and an epidemiologist. RoB assessments were compared to the reference standard by the experienced information specialist, and the number of disagreements with the reference standard were counted. A two-level deviation in the domain rating (eg, a high RoB rating instead of a low RoB rating) was counted as an error. A single-level deviation in the domain rating (eg, unclear RoB instead of low RoB) was recorded as a difference of opinion.

\section{Results}

The SR and comparison study began in August 2017. The comparison study was completed at the end of March 2018, while the SR was published in October 2018 [19].

\section{Time Taken to Conduct Systematic Review Tasks}

The approximate time taken for the manual team to produce a draft of the background, methods, and results sections (ie, 16 SR tasks) was 126 hours (Table 5). Approximately 101 hours were spent doing all the tasks, and approximately 25 hours were spent learning about the tasks. For the SR tasks where times were compared (ie, SR Tasks 5-9 and 12), the total time taken by the manual team was 41 hours and 33 minutes. The time spent doing the SR tasks was 35 hours and 28 minutes, while the time spent learning about the SR tasks was 6 hours and 5 minutes. The automation team took 11 hours and 48 minutes to complete all the SR tasks. The time spent doing the SR tasks was 10 hours and 30 minutes, while the time spent learning about the SR tasks was 1 hour and 18 minutes (Table 5). The times spent on Task 12: Assess the RoB were not equivalent, as the RobotReviewer tool only partially automates the task. It assessed RoB in four of the seven domains, while the manual team assessed RoB in seven of the seven RoB domains. 
Table 5. Time taken for the manual and automation teams to learn and complete each systematic review (SR) task.

\begin{tabular}{|c|c|c|c|c|c|c|c|}
\hline \multirow[t]{2}{*}{$\begin{array}{l}\text { SR task } \\
\text { No. }\end{array}$} & \multirow[t]{2}{*}{ SR task } & \multicolumn{2}{|c|}{$\begin{array}{l}\text { Total time, } \\
\text { hours:minutes }\end{array}$} & \multicolumn{2}{|c|}{$\begin{array}{l}\text { Time doing task, } \\
\text { hours:minutes }\end{array}$} & \multicolumn{2}{|c|}{$\begin{array}{l}\text { Time learning task, } \\
\text { hours:minutes }\end{array}$} \\
\hline & & Manual & Automation & Manual & Automation & Manual & Automation \\
\hline 1 & Formulate the question & $1: 00^{\mathrm{a}}$ & $\mathrm{N} / \mathrm{A}^{\mathrm{b}}$ & $1: 00^{\mathrm{a}}$ & N/A & $0: 00$ & N/A \\
\hline 2 & Check for similar reviews & $1: 00^{\mathrm{a}}$ & N/A & $1: 00^{\mathrm{a}}$ & N/A & $0: 00$ & N/A \\
\hline 3 & Write the protocol & $4: 00^{\mathrm{a}}$ & N/A & $4: 00^{\mathrm{a}}$ & N/A & $0: 00$ & N/A \\
\hline 4 & Design the systematic search & $13: 00^{\mathrm{a}}$ & N/A & $13: 00^{\mathrm{a}}$ & N/A & $0: 00$ & N/A \\
\hline 5 & Run the systematic search & $6: 15$ & $1: 20$ & $5: 00$ & $0: 37$ & $1: 15$ & $0: 43$ \\
\hline 6 & Deduplicate the search results & $2: 09$ & $0: 36$ & $2: 09$ & $0: 12$ & 0:00 & $0: 24$ \\
\hline 7 & Screen the titles and abstracts & $5: 10$ & $3: 33$ & $4: 40$ & $3: 28$ & $0: 30$ & $0: 05$ \\
\hline 8 & Find the full text & $0: 50$ & $0: 23$ & $0: 50$ & $0: 18$ & 0:00 & $0: 05$ \\
\hline 9 & Screen the full text & $3: 29$ & $3: 44$ & $3: 29$ & $3: 44$ & $0: 00$ & $0: 00$ \\
\hline 10 & Conduct a citation analysis & $7: 43$ & N/A & $7: 43$ & N/A & 0:00 & N/A \\
\hline 11 & Extract the data & $9: 42$ & N/A & $9: 42$ & N/A & $0: 00$ & N/A \\
\hline 12 & Assess the risk of bias & $23: 40$ & $2: 12^{c}$ & $19: 20$ & $2: 11^{\mathrm{c}}$ & $4: 20$ & $0: 01^{\mathrm{c}}$ \\
\hline 13 & Synthesize the data & $10: 00$ & N/A & $8: 00$ & N/A & $2: 00$ & N/A \\
\hline 14 & Rerun the systematic search & $0: 22$ & N/A & $0: 22$ & N/A & $0: 00$ & N/A \\
\hline 15 & Conduct a meta-analysis & $16: 00$ & N/A & $10: 00$ & N/A & $6: 00$ & N/A \\
\hline 16 & Write the results & $21: 20$ & N/A & $10: 40$ & N/A & $10: 40$ & N/A \\
\hline All tasks & Tasks done by both teams & $41: 33$ & $11: 48$ & $35: 28$ & $10: 30$ & $6: 05$ & $1: 18$ \\
\hline All tasks & Tasks done by manual team & $125: 40^{\mathrm{a}}$ & N/A & $100: 55^{\mathrm{a}}$ & N/A & $24: 45^{\mathrm{a}}$ & N/A \\
\hline
\end{tabular}

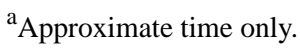

${ }^{b}$ N/A: not applicable; task not done by automation team.

${ }^{\mathrm{c}}$ Task partially completed; four of seven domains assessed.

\section{Quality of the Systematic Review Tasks}

The manual team had more errors in Task 5: Run the systematic search, with eight types of errors made compared to three by the automation team. Regarding Task 12: Assess the RoB, the manual team had a total of 25 differences in opinion from the reference standard compared to only 20 from the automation

team. The manual team had fewer errors in Task 6: Deduplicate the search results by identifying all duplicates while excluding one unique study, compared to the automation team who missed seven duplicates and removed two unique studies. The teams performed similarly for both SR screening tasks (ie, Tasks 7 and 9) (Table 6). 
Table 6. Quality indicators of each task in the systematic review (SR) process.

\begin{tabular}{lllll}
\hline SR task No. & SR task & Evaluation criteria & Manual team, $\mathrm{n}$ & Automation team, $\mathrm{n}$ \\
\hline 5 & Run the systematic search & Number of different types of errors made & 8 & 3 \\
6 & Deduplicate the search results & Number remaining after deduplication & 586 & 594 \\
6 & Deduplicate the search results & Unique studies removed & 1 & 2 \\
6 & Deduplicate the search results & Duplicates missed & 0 & 3 \\
7 & Screen the titles and abstracts & Studies included & 38 & 0 \\
7 & Screen the titles and abstracts & Relevant studies excluded & 0 & - \\
8 & Find the full text & Full texts ordered from library & $-{ }^{\mathrm{a}}$ & 22 \\
9 & Screen the full text & Studies included & 30 & 0 \\
9 & Screen the full text & Relevant studies excluded & 0 & 36 \\
12 & Assess the risk of bias & Same domain & 31 & 20 \\
12 & Assess the risk of bias & Different domain & Errorss in domain & 0 \\
\hline
\end{tabular}

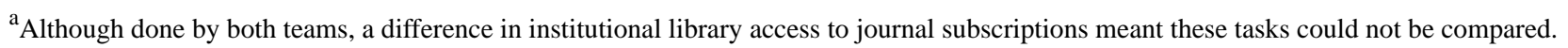

\section{Availability of Data and Materials}

The data sets used and/or analyzed during this study are available from the corresponding author on reasonable request.

\section{Discussion}

\section{Principal Findings}

To complete a draft of the background, methods, and results of the SR, the manual team took approximately 126 hours. To complete the six SR tasks evaluated in this study, the manual team took approximately 42 hours while the automation team took 12 hours. This equates to potential time savings of 30 hours. Due to the small amount of time taken to learn how to use the SRA tools (ie, 2 hours), the time required to learn how to use SRA tools should not be a barrier to their uptake among novice researchers. Regarding methodological quality of SR tasks done with SRA tools, we found that the error rates between teams was minimal and would not significantly impact on the quality of the SR. The manual team had more errors in two of the SR tasks (Tasks 5 and 12) and fewer errors in one SR task (Task $8)$; neither team had errors in two of the SR tasks (Tasks7 and 9).

The automation team was faster in five of six of the SR tasks compared in this study, where the increased speed of four of the tasks was due to an improvement on a manual process. For instance, to modify search strings, researchers may use the replace tool in Microsoft Word to manually change the database syntax, or they may use a drag-and-drop process when screening in EndNote. This replacing of manual, tedious work with an SRA tool is an obvious benefit of automation. The other SR task where the automation team was faster was the RoB assessment. It is important to note that although the time reduction for assessing RoB was substantial in the automation team, this team only assessed four out of the seven domains while the manual team assessed all seven of them. The only SR task where the manual team was faster was Task 8: Screen the full text, although the times were similar (209 to 224 minutes; a difference of 7.5 minutes per researcher). This was most likely due to the SR task requiring the reading and comprehension of articles to determine if they were eligible; in this case, the manual team members were more experienced as the SR was on a topic of their expertise. This suggests that for SR tasks where the interpretation or understanding of information plays a major role, there are lessened potential time savings for SRA tools.

The total time difference between the manual team and the automation team was substantial and could be translated to significant cost savings in funded studies. The savings may be attributable to several factors. Due to variations within the novice researchers' experience (0-3 SRs each), it is likely that the time savings were due in part to participant experience. A lack of blinding and randomization may have contributed bias, where the automation team could have pushed themselves to finish the SR tasks faster than they would under normal circumstances. However, due to the vast time difference between groups and both groups being novice users, it is clear that the SRA tools were the primary contributor to the time savings. This finding has been confirmed in other studies. In an RCT, an SRA tool was found to speed up the translation of search strings across databases by $25 \%$, or 15 minutes, per database [8]. A test of three different screening tools found time savings of 154 to 185 hours for a fully automated approach and 61 to 92 hours for a semi-automated approach [24]. Another test of an automated screening tool on three SRs found a 50\% reduction in screening workload in two of the SRs and a $40 \%$ reduction in the third [25]. Findings from this study align more with the findings of Wallace et al [25], with time savings between $25 \%$ and $50 \%$. Further research is required to replicate and confirm the findings from this study in novice researchers to better understand the estimated time savings produced by SRA tools.

As all participants were novice users of the automation tools, the process to learn a new SRA tool may be comparable to the manual team learning to complete a new SR task. Although the 
availability of training and support for the SRA tools would have reduced the time spent learning to use them, similar SR training and support is routinely available at universities for standard manual methods.

It currently takes a long time for an SR to go from conception to publication (mean 67.3 weeks) [5]. A recent case study looking at time logs across 12 simulated SRs found the average time to complete an SR (mean 3821 records screened; 20 studies included) was 463 days (66 weeks) and 881 person-hours [26]. Individual tasks required were selecting studies (229 hours, $26 \%$ ), collecting data (211 hours, 24\%), preparing the report (202 hours, 23\%), conducting the meta-analysis (149 hours, $17 \%$ ), and descriptive synthesis (52 hours, 6\%) [26]. The SR used in this study [19] was substantially smaller (586 records screened; 16 studies included) and less time was required, but the percentage of time spent on comparable tasks generally aligned: selecting studies consumed 39 person-hours (31\%), collecting data consumed 43 hours $(35 \%)$, preparing the report consumed 26 hours $(20 \%)$, and conducting the meta-analysis consumed 16 hours $(12 \%)$.

The total time and person-hours from conception to publication is still substantial for SRs that employ SRA tools [26]. A recent case study found that by focusing on a single SR, using SRA tools, and having experienced reviewers, a medium-sized SR of RCTs (1381 records screened; 8 studies included) could be submitted for publication within 16 calendar days (10 working days; 66 person-hours) from conception [21]. This case study also highlights a significant difference between the findings in a novice versus experienced researcher team already familiar with the tools. However, the topics in the experienced case study and in this case study were different; in addition, further research is required to compare novice and experienced teams' performance on the same topic for firmer estimates of time and error rates to be obtained. Despite the topic difference, this case study had similarities in that it was a medium-sized review and it only included RCTs.
In the case study completed by the experienced reviewers, approximately 17 hours were required to conduct the six tasks that were completed by the automation team in this study, who took approximately 12 hours. Although the cases are not directly comparable, this suggests that while the experience of the researcher team is relevant, it is likely only a small driver of the time savings.

\section{Limitations and Strengths of the Study}

This study was limited by its case study design, with only a single SR used in the comparison as well as variation in the experience of the novice researchers. The times estimated for Tasks 1 to 4 of the study have less reliability compared to other steps, which should be considered when interpreting findings. The study was limited by the assessment of each SR task individually, outside of the context of the entire SR, which makes results harder to apply to a full SR done with SRA tools. Additionally, due to the niche nature of the research question, the number of studies identified by the search strategy was small compared to other SRs in health; this may have implications for generalizing to other SRs the overall time required to complete the review. Further, this case study was not registered in a trial or study registry database. A strength of the study is that the time measured was the time that each person engaged in active SR tasks, with breaks excluded from the reported time. Another strength is that the time spent learning about the SR tasks was recorded independently from the time spent doing the tasks. The final strength is that the SR used was a real research project, which means the impact of SRA tools can be shown in a real-world setting.

\section{Conclusions}

For the majority of SR tasks where an SRA tool was used, the time required to complete that task was reduced for novice researchers while methodological quality was maintained. Further research is required to confirm these findings.

\section{Acknowledgments}

The authors would like to thank Chris Del Mar and Elaine Beller for help with the design of the study, Anna Mae Scott for help with assessing the RoB of the studies and for feedback on the manuscript, David Honeyman for help with assessing the quality of the translations of the search strings, Mina Bhakit for feedback on the manuscript, and Katrina Campbell for help with the design of the study and feedback on the manuscript. This research did not receive any specific grant from funding agencies in the public, commercial, or not-for-profit sectors.

\section{Authors' Contributions}

JC, CM, and SM were responsible for study conception and design. CM, GC, CR, and SM were responsible for acquisition of the data. JC, GC, and SM were responsible for analysis and/or interpretation of the data. JC and SM were responsible for drafting of the manuscript. JC, CM, GC, CR, and SM were responsible for critical revision of the manuscript.

\section{Conflicts of Interest}

JC declares that he is a developer of some of the tools used in this study and has won prize money from the Australian Library Information Association to continue development of these tools.

\section{Multimedia Appendix 1}

Supplementary Table S1: Marking criteria for errors in search string translations. 


\section{References}

1. Coleman K, Norris S, Weston A, Grimmer-Somers K, Hillier S, Merlin T, et al. NHMRC Additional Levels of Evidence and Grades for Recommendations for Developers of Guidelines. Canberra, Australia: National Health and Medical Research Council (NHMRC); 2009. URL: https://www.mja.com.au/sites/default/files/NHMRC.levels.of.evidence.2008-09.pdf [accessed 2021-05-11]

2. Higgins JPT, Green S. Cochrane Handbook for Systematic Reviews of Interventions. Version 5.1.0. London, UK: The Cochrane Collaboration; 2011.

3. Tsertsvadze A, Chen Y, Moher D, Sutcliffe P, McCarthy N. How to conduct systematic reviews more expeditiously? Syst Rev 2015 Nov 12;4:160 [FREE Full text] [doi: 10.1186/s13643-015-0147-7] [Medline: 26563648]

4. Créquit P, Trinquart L, Yavchitz A, Ravaud P. Wasted research when systematic reviews fail to provide a complete and up-to-date evidence synthesis: The example of lung cancer. BMC Med 2016 Jan 20;14:8 [FREE Full text] [doi:

10.1186/s12916-016-0555-0] [Medline: 26792360]

5. Borah R, Brown AW, Capers PL, Kaiser KA. Analysis of the time and workers needed to conduct systematic reviews of medical interventions using data from the PROSPERO registry. BMJ Open 2017 Feb 27;7(2):e012545 [FREE Full text] [doi: 10.1136/bmjopen-2016-012545] [Medline: 28242767]

6. Khangura S, Konnyu K, Cushman R, Grimshaw J, Moher D. Evidence summaries: The evolution of a rapid review approach. Syst Rev 2012 Feb 10;1:10 [FREE Full text] [doi: 10.1186/2046-4053-1-10] [Medline: 22587960]

7. Harker J, Kleijnen J. What is a rapid review? A methodological exploration of rapid reviews in Health Technology Assessments. Int J Evid Based Healthc 2012 Dec;10(4):397-410. [doi: 10.1111/j.1744-1609.2012.00290.x] [Medline: $\underline{23173665]}$

8. Clark JM, Sanders S, Carter M, Honeyman D, Cleo G, Auld Y, et al. Improving the translation of search strategies using the Polyglot Search Translator: A randomized controlled trial. J Med Libr Assoc 2020 Apr;108(2):195-207 [FREE Full text] [doi: 10.5195/jmla.2020.834] [Medline: 32256231]

9. Rathbone J, Carter M, Hoffmann T, Glasziou P. Better duplicate detection for systematic reviewers: Evaluation of Systematic Review Assistant-Deduplication Module. Syst Rev 2015 Jan 14;4:6 [FREE Full text] [doi: 10.1186/2046-4053-4-6] [Medline: 25588387]

10. Ouzzani M, Hammady H, Fedorowicz Z, Elmagarmid A. Rayyan-A web and mobile app for systematic reviews. Syst Rev 2016 Dec 05;5(1):210 [FREE Full text] [doi: 10.1186/s13643-016-0384-4] [Medline: 27919275]

11. Covidence. Melbourne, Australia: Veritas Health Innovation; 2019. URL: https://www.covidence.org/ [accessed 2021-05-03]

12. Marshall IJ, Kuiper J, Wallace BC. RobotReviewer: Evaluation of a system for automatically assessing bias in clinical trials. J Am Med Inform Assoc 2016 Jan;23(1):193-201 [FREE Full text] [doi: 10.1093/jamia/ocv044] [Medline: 26104742]

13. Beller E, Clark J, Tsafnat G, Adams C, Diehl H, Lund H, Founding members of the ICASR group. Making progress with the automation of systematic reviews: Principles of the International Collaboration for the Automation of Systematic Reviews (ICASR). Syst Rev 2018 May 19;7(1):77 [FREE Full text] [doi: 10.1186/s13643-018-0740-7] [Medline: 29778096]

14. van Altena AJ, Spijker R, Olabarriaga SD. Usage of automation tools in systematic reviews. Res Synth Methods 2019 Mar;10(1):72-82. [doi: 10.1002/jrsm.1335] [Medline: 30561081]

15. O'Connor AM, Tsafnat G, Gilbert SB, Thayer KA, Wolfe MS. Moving toward the automation of the systematic review process: A summary of discussions at the second meeting of International Collaboration for the Automation of Systematic Reviews (ICASR). Syst Rev 2018 Jan 09;7(1):3 [FREE Full text] [doi: 10.1186/s13643-017-0667-4] [Medline: 29316980]

16. O'Connor AM, Tsafnat G, Gilbert SB, Thayer KA, Shemilt I, Thomas J, et al. Still moving toward automation of the systematic review process: A summary of discussions at the third meeting of the International Collaboration for Automation of Systematic Reviews (ICASR). Syst Rev 2019 Feb 20;8(1):57 [FREE Full text] [doi: 10.1186/s13643-019-0975-y] [Medline: 30786933]

17. von Elm E, Altman DG, Egger M, Pocock SJ, Gøtzsche PC, Vandenbroucke JP, STROBE Initiative. The Strengthening the Reporting of Observational Studies in Epidemiology (STROBE) statement: Guidelines for reporting observational studies. J Clin Epidemiol 2008 Apr;61(4):344-349. [doi: 10.1016/j.jclinepi.2007.11.008] [Medline: 18313558]

18. Moher D, Liberati A, Tetzlaff J, Altman DG, PRISMA Group. Preferred reporting items for systematic reviews and meta-analyses: The PRISMA statement. BMJ 2009 Jul 21;339:b2535 [FREE Full text] [doi: 10.1136/bmj.b2535] [Medline: 19622551]

19. McFarlane C, Ramos CI, Johnson DW, Campbell KL. Prebiotic, probiotic, and synbiotic supplementation in chronic kidney disease: A systematic review and meta-analysis. J Ren Nutr 2019 May;29(3):209-220. [doi: 10.1053/j.jrn.2018.08.008] [Medline: 30366767]

20. Cleo G, Scott AM, Islam F, Julien B, Beller E. Usability and acceptability of four systematic review automation software packages: A mixed method design. Syst Rev 2019 Jun 20;8(1):145 [FREE Full text] [doi: 10.1186/s13643-019-1069-6] [Medline: 31221212]

21. Clark J, Glasziou P, Del Mar C, Bannach-Brown A, Stehlik P, Scott AM. A full systematic review was completed in 2 weeks using automation tools: A case study. J Clin Epidemiol 2020 May;121:81-90. [doi: 10.1016/j.jclinepi.2020.01.008] [Medline: $\underline{32004673]}$ 
22. Tsafnat G, Glasziou P, Choong MK, Dunn A, Galgani F, Coiera E. Systematic review automation technologies. Syst Rev 2014 Jul 09;3:74 [FREE Full text] [doi: 10.1186/2046-4053-3-74] [Medline: 25005128]

23. Higgins JPT, Altman DG, Gøtzsche PC, Jüni P, Moher D, Oxman AD, Cochrane Bias Methods Group, Cochrane Statistical Methods Group. The Cochrane Collaboration's tool for assessing risk of bias in randomised trials. BMJ 2011 Oct 18;343:d5928 [FREE Full text] [doi: 10.1136/bmj.d5928] [Medline: 22008217]

24. Gates A, Guitard S, Pillay J, Elliott S, Dyson M, Newton A, et al. Performance and usability of machine learning for screening in systematic reviews: A comparative evaluation of three tools. Syst Rev 2019 Nov 15;8(1):278 [FREE Full text] [doi: 10.1186/s13643-019-1222-2] [Medline: 31727150]

25. Wallace BC, Trikalinos TA, Lau J, Brodley C, Schmid CH. Semi-automated screening of biomedical citations for systematic reviews. BMC Bioinformatics 2010 Jan 26;11:55 [FREE Full text] [doi: 10.1186/1471-2105-11-55] [Medline: 20102628]

26. Pham B, Bagheri E, Rios P, Pourmasoumi A, Robson RC, Hwee J, et al. Improving the conduct of systematic reviews: A process mining perspective. J Clin Epidemiol 2018 Nov;103:101-111. [doi: 10.1016/j.jclinepi.2018.06.011] [Medline: 30297037]

\author{
Abbreviations \\ ICASR: International Collaboration for the Automation of Systematic Reviews \\ PRISMA: Preferred Reporting Items for Systematic Reviews and Meta-Analyses \\ RCT: randomized controlled trial \\ RoB: risk of bias \\ SARA: System for Automatically Requesting Articles \\ SR: systematic review \\ SRA: systematic review automation \\ SRA-Helper: Systematic Review Accelerator Helper \\ STROBE: Strengthening the Reporting of Observational Studies in Epidemiology
}

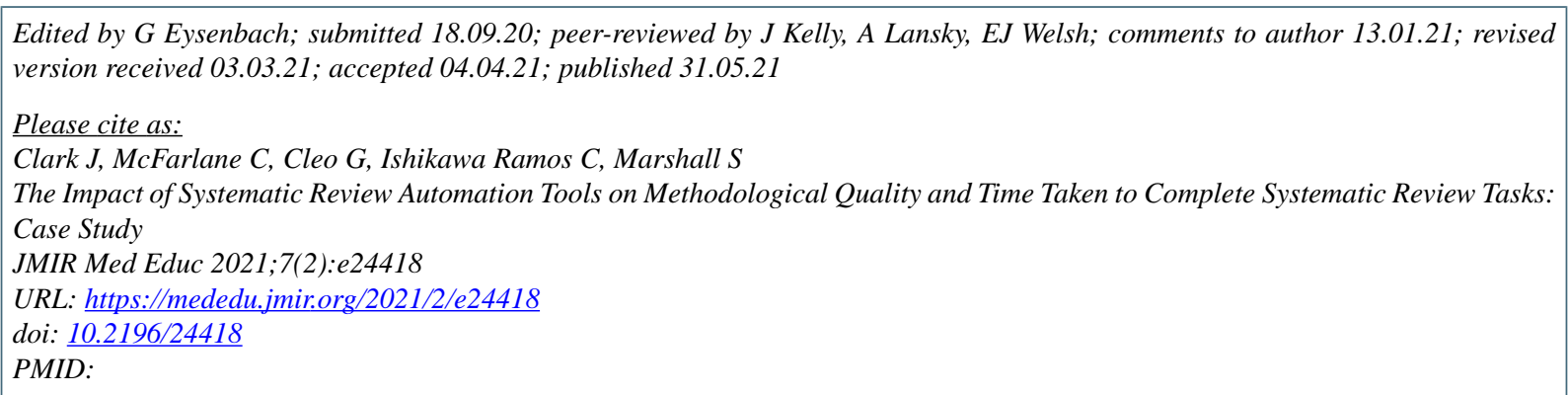

CJustin Clark, Catherine McFarlane, Gina Cleo, Christiane Ishikawa Ramos, Skye Marshall. Originally published in JMIR Medical Education (https://mededu.jmir.org), 31.05.2021. This is an open-access article distributed under the terms of the Creative Commons Attribution License (https://creativecommons.org/licenses/by/4.0/), which permits unrestricted use, distribution, and reproduction in any medium, provided the original work, first published in JMIR Medical Education, is properly cited. The complete bibliographic information, a link to the original publication on https://mededu.jmir.org/, as well as this copyright and license information must be included. 